\title{
IL-10 therapy in Crohn's disease: at the crossroads
}

\section{H Herfarth, J Schölmerich}

\section{Treatment of Crohn's disease with the anti-inflammatory cytokine interleukin 10}

n nterleukin 10 (IL-10) was initially discovered and isolated on the basis of its ability to suppress cytokine synthesis by Thl helper cells. Macrophages and their secreted mediators are the primary target of IL-10. IL-10 downregulates expression of class II and B7 molecules, as well as IL- 12 production, thus impairing the macrophage dependent stimulation of antigen reactive Th- 1 cells.

The important regulatory role of IL-10 in the gut became obvious when IL-10 deficient mice (IL-10 $0^{-/}$), generated by gene targeting, developed chronic enterocolitis. ${ }^{2}$ More interestingly, IL-10 mice kept under germfree conditions do not develop enterocolitis, which suggests that in the absence of the immunomodulatory effects of IL-10, an unrestricted intestinal inflammatory response develops towards normal enteric antigens. The observations in the IL- $10^{-/-}$mice lay the foundation for administration of IL-10 in several animal models. The results of these studies clearly showed prevention of intestinal inflammation by IL-10, mainly by downregulation of an intestinal proinflammatory Thl response. However, systemic IL-10 administration was successful only when administered prior to the initiation of colitis but was ineffective at reversing any established inflammation. ${ }^{34}$

\section{IL-10: THE CLINICAL EXPERIENCE}

Based on the successful experimental findings in animal models of intestinal inflammation, IL-10 therapy was introduced as a potential new antiinflammatory therapy in Crohn's disease (CD). Several large multicentre trials were performed testing multiple IL-10 dosages in patients with mild/moderate or therapy refractory $\mathrm{CD}$, as well as in patients undergoing curative ileal or ileocolonic resection to prevent endoscopic postoperative occurrence by systemic administration. ${ }^{5-7}$ All data indicate that IL-10 therapy is safe and well tolerated. But IL-10 treatment did not result in significantly higher remission rates or clinical improvement compared with placebo treatment. There are several explanations for the disappointment with this therapeutic strategy: (i) With the administered dose of IL-10 in the clinical trials, the ultimate local IL-10 concentrations in the intestine could be too low to result in downregulation of inflammation. Unfortunately, increasing the dose of systemically administered IL-10 is limited due to side effects (for example, anaemia, headache).

(ii) IL-10 administration is only successful for preventing and not treating an established disease, as was suggested by the results of the animal experiments.

(iii) Administration of IL-10 alone fails to effectively suppress the dysregulation of the wide variety of proinflammatory mediators that are involved in the perpetuation of chronic intestinal inflammation.

(iv) The immunostimulatory properties of IL-10 on B cells and on interferon $\gamma$ (IFN- $\gamma$ ) production by $\mathrm{CD}^{+}, \mathrm{CD}^{+}$, and/or natural killer cells counterbalance its immunosuppressive properties.

In this issue of Gut, data are presented which may explain, at least in part, the dilemma of IL-10 therapy in CD [see page 191] ${ }^{8}$ Tilg et al have investigated the influence of subcutaneous administration of various doses of human recombinant IL-10 on lymphocytic IFN- $\gamma$ production and lipopolysaccharide (LPS) induced tumour necrosis factor (TNF) secretion by macrophages in whole blood assays as well as on serum neopterin and nitrite/ nitrate levels. The study was conducted using samples from two multicentre therapeutic trials in patients with steroid dependent chronic active $\mathrm{CD}$ and patients with mild to moderately active CD.

In patients treated with the highest dose of IL-10 $(20 \mu \mathrm{g} / \mathrm{kg})$, the study described a significant increase in neopterin, which is produced by human monocytes/macrophages in response to IFN- $\gamma$, as well as an increase in phytohaemagglutinin induced IFN- $\gamma$ production compared with pretreatment levels. Furthermore, LPS induced TNF- $\alpha$ production was dose dependently downregulated by IL-10. Neither the elevation in neopterin or IFN- $\gamma$ nor suppression of TNF correlated with the clinical response of the patients, which may also reflect the divergence of the clinical (Crohn's disease activity index) and immunological (for example, proinflammatory mediators) readouts in trials employing cytokine or anticytokine strategies.

The immunopotentiating effects of IL-10 found by Tilg et al are corroborated by a study in healthy volunteers subjected to experimental endotoxaemia. Systemic IL-10 treatment enhanced endotoxin (LPS) induced IFN- $\gamma$ release as well as the IFN- $\gamma$ dependent chemokines IFN- $\boldsymbol{\gamma}$ inducible protein 10 (IP-10) and monokine induced by IFN- $\gamma$ (MIG). The stimulatory effects were most pronounced when IL-10 administration was performed one hour after the LPS challenge.

\section{IL-10 THERAPY IN CD: A DEAD END?}

What then are the lessons to be learned from the experimental and clinical experiences with IL-10?

The clinical studies published so far clearly indicate no relevant advantage of systemic IL-10 therapy compared with placebo in active and postoperative CD. Furthermore, as the results of Tilg et al indicate, higher doses of systemically administered IL-10 (which were also used in the clinical trials) may be detrimental rather than helpful. Nevertheless, the concept of rebalancing the intestinal immunological homeostasis with IL-10 is still very compelling and applying IL-10 locally in high concentrations may result in strong immunosuppression and circumvent the systemic side effects. So far we do not know whether high IL-10 concentrations also have immunostimulatory properties in the intestine as the study of Tilg et al was performed using only whole blood or serum. Furthermore, as indicated above, IL-10 prevented intestinal inflammation in animal studies but could never completely cure an established disease, indicating that IL-10 therapy in CD would succeed rather in preventing relapses than abolishing acute or chronic inflammation.

Recently, a novel compelling approach of local IL-10 therapy, which could also be used as a long term therapeutic approach, has been described. Steidler et al demonstrated that intragastric administration of an IL-10 secreting Lactobacillus lactis, generated by genetic engineering, caused a significant reduction in colitis in two different mouse models. ${ }^{10}$ Thus with dietary supplementation it may be possible to deliver high concentrations of IL-10 within the gut, thus preventing the recurrence of CD. However, this work has just proved a therapeutic principle and there is still a long way to go until such a concept can be evaluated in clinical studies.

Gut 2002;50: 146-147 
Authors' affiliations

H Herfarth, J Schölmerich, Department of Internal Medicine I, University of Regensburg,

Regensburg, Germany

Correspondence to: Dr H Herfarth, Klinik und Poliklinik für Innere Medizin I, Klinikum der Universität Regensburg, 93042 Regensburg, Germany;

hans.herfarth@klinik.uni-regensburg.de

\section{REFERENCES}

1 Mosmann TR. Properties and functions of interleukin-10. Adv Immunol 1994:56:1-16.

2 Rennick DM, Fort MM. Lessons from genetically engineered animal models. XII. IL-10-deficient (IL-10(-/-) mice and intestinal inflammation. Am J Physiol Gastrointest Liver Physiol 2000;278:G829-33.

3 Herfarth HH, Böcker U, Janardhanam R, ef al. Subtherapeutic corticosteroids potentiate the ability of interleukin-10 to prevent chronic inflammation in rats. Gastroenterology $1998 ; 115: 856-65$

4 Barbara G, Xing Z, Hogaboam CM, et al. Interleukin 10 gene transfer prevents experimental colitis in rats. Gut 2000;46:344-9.

5 Fedorak RN, Gangl A, Elson CO, et al. Recombinant human interleukin 10 in the treatment of patients with mild to moderately active Crohn's disease. The Interleukin 10 Inflammatory Bowel Disease Cooperative Study Group. Gastroenterology 2000;119:1473-82

6 Schreiber S, Fedorak RN, Nielsen $\mathrm{OH}$, et al. Safety and efficacy of recombinant human interleukin 10 in chronic active Crohn's disease. Crohn's Disease IL-10 Cooperative Study Group. Gastroenterology

2000;119:1461-72.

7 Colombel JF, Rutgeerts P, Malchow $\mathrm{H}$ et al. Interleukin 10 (Tenovil) in the prevention of postoperative recurrence of Crohn's disease. postoperative recurre

8 Tilg $\mathbf{H}$, van Montfrans $C$, van den Ende A, ef al. Treatment of Crohn's disease with recombinant human interleukin 10 induces the proinflammatory cytokine interferon gamma. Gut 2002;50:191-5.

9 Lauw FN, Pajkrt D, Hack CE, et al. Proinflammatory effects of IL-10 during human endotoxemia. J Immunol 2000; 165:2783-9.

10 Steidler L, Hans W, Schotte L, et al. Treatment of murine colitis by Lactococcus lactis secreting interleukin-10. Science 2000;289: 1352-5. 
Inflammatory bowel disease

\section{Life and death in the gut: more killing, less Crohn's}

\section{A Sturm, C Fiocchi}

\section{The beneficial effects of infliximab, the tumour necrosis factor antibody, in Crohn's disease may be mediated by apoptosis of activated mucosal T cells}

T he advent of new biological agents for the treatment of autoimmune and chronic inflammatory disorders is drastically altering the approach to management while setting higher standards for therapeutic expectations. Only a fraction of the new biological agents keeps the promise of improved efficacy and specificity but the few that do can generate impressive results as we are currently witnessing for anti-tumour necrosis factor (TNF)- $\alpha$ therapy in rheumatoid arthritis and Crohn's disease. ${ }^{12}$ Based on these results, a number of other conditions where TNF- $\alpha$ biological activity may play a pathogenic role, such as psoriasis, sarcoidosis, spondyloarthropathy, Behçet's syndrome, and sepsis, are being treated using TNF- $\alpha$ blocking antibodies with variable but generally positive results. Since the first report of the use of infliximab in human disease, the literature has swelled to over 200 publications on practical applications and theoretical considerations of this humanised antibody. In this enormous body of information however, disappointingly little is found on the mechanisms of action of infliximab. Almost invariably the optimism caused by the feeling of finally having discovered a magic bullet against unyielding diseases causes all interest and resources to be shifted to more clinical trials. Although this reaction is understandable, all too often it comes at the expense of investigating mechanisms of action that would ultimately lead to a safer and more reliable use of the biological agent, or even the discovery of better biologicals. Thus the study of ten Hove et al in this issue of Gut, describing induction of mucosal $\mathrm{T}$ cell apoptosis during infliximab treatment of Crohn's disease, is a welcome and necessary complement to our still incomplete knowledge of the effect and manipulation of TNF- $\alpha$ in chronic intestinal inflammation [see page 206]. ${ }^{3}$

The in vivo action of infliximab has been more extensively explored in rheumatoid arthritis where blocking of TNF- $\alpha$ alters production of interleukin (IL)-6, IL-8, monocyte chemotactic protein 1 , vascular endothelial growth factor, matrix metalloproteinases 1 and 3, angiogenesis, and the recruitment of inflammatory cells. ${ }^{4}$ Targeting of these and other activities is also of obvious importance in Crohn's disease in view of the broad role of TNF- $\alpha$ in mucosal inflammation. ${ }^{5}$ Unfortunately, except for the demonstration of a reduction in $\mathrm{CD}^{+}, \mathrm{CD}^{+} \mathrm{T}$ cells, and $\mathrm{CD}^{+} 8^{+}$monocytes, downregulation of cell adhesion molecules, and decrease in IL-4 and TNF- $\alpha^{+}$cells in the gut, little else has been published on the cellular and molecular effects of infliximab in patients with Crohn's disease. ${ }^{6}$ The aetiopathogenesis of Crohn's disease is still uncertain but there is good evidence to indicate that this condition falls into the category of disease associated with defective $\mathrm{T}$ cell apoptosis, a fundamental mechanism of immune homeostasis indispensable to the maintenance of health. ${ }^{7}$ Without proper control of apoptosis, the complex process regulating proliferation and death of naive and memory $\mathrm{T}$ cells during an immune response goes awry, and an inappropriate accumulation of $\mathrm{T}$ cells ensues in the tissues and leads to inflammation. ${ }^{8}$ This deleterious series of events appears to occur in Crohn's disease, as indicated by studies showing that mucosal $\mathrm{T}$ cells are resistant to multiple apoptotic stimuli and have a reduced expression of the proapoptotic Bax protein, while an imbalance between Bax and the antiapoptotic $\mathrm{Bcl}-2$ protein is present in the inflamed mucosa. ${ }^{9-11}$ Therefore, it is reasonable to assume that eliminating excessive T cells could restore the gut to its normal state of physiological inflammation or, at least, a state of controlled inflammation (fig 1). Strong evidence for this effect is provided by animal models where experimental colitis is abrogated by induction of increased $\mathrm{T}$ cell apoptosis with IL-12 antibodies, blockade of IL-6 trans signalling, or deletion of $\mathrm{CD} 44 \mathrm{v} 7^{+}$ cells. ${ }^{12-14}$

Based on the above reasoning and experimental evidence, ten Hove et al hypothesised that infliximab, in addition to neutralising soluble TNF- $\alpha$, could improve Crohn's disease by inducing apoptosis of mucosal T cells. ${ }^{3}$ To test this hypothesis, the authors measured mark- ers of activation and cell death in peripheral and mucosal $\mathrm{T}$ cells of patients with clinically active Crohn's disease receiving a therapeutic infusion of infliximab. In patients with a clinical response they found only minor changes in the properties and apoptosis of circulating $\mathrm{T}$ cells while the number of apoptotic cells, primarily $\mathrm{CD}^{+} \mathrm{T}$ cells, significantly increased in mucosal biopsies taken 24 hours after the start of treatment. They complemented these observations by demonstrating that infliximab could induce in vitro apoptosis of activated but not resting Jurkat $\mathrm{T}$ cells. As mucosal T cells in active Crohn's disease are in an enhanced state of activation, the authors concluded that the beneficial effects of infliximab may be mediated by killing of activated mucosal $\mathrm{T}$ cells (fig $1)$. This conclusion is warranted even though in vitro studies on infliximab mediated apoptosis of resting and activated peripheral blood and lamina propria $\mathrm{T}$ cells were not performed. The results could have reinforced the conclusion reached by the authors, and shed some light on whether defective apoptosis in Crohn's disease is an intrinsic systemic defect or one that is only detectable on exposure of $\mathrm{T}$ cells to the immunological challenges of the mucosa. ${ }^{15}$

A number of interesting issues, questions, and speculations are raised by this work. For starters, as ten Hove et al point out, the exact mechanism of infliximab mediated killing of mucosal $\mathrm{T}$ cells remains to be explored, especially knowing that apoptosis is not induced by direct in vitro exposure of these cells to TNF- $\alpha .^{10}$ Is induction of mucosal T cell apoptosis the only mechanism responsible for the beneficial effects of infliximab? Most likely not in view of the multiplicity of biological activities of TNF- $\alpha$ and this antibody. ${ }^{45}$ Whether induction of apoptosis is the dominant mechanism of action should be ascertained in the near future once studies similar to the one reported in this issue of Gut are repeated in other diseases that also benefit from TNF- $\alpha$ blockade. Finally, if indeed killing of activated $\mathrm{T}$ cells is the modus operandi of infliximab, this could have broad therapeutic implications. In fact, any condition characterised by increased numbers of activated $\mathrm{T}$ cells may profit from killing of these cells in the affected organs. There is preliminary evidence that infliximab provides clinical benefit for some patients with steroid refractory ulcerative colitis, ${ }^{16}$ which is also characterised by high numbers of activated T cells in the mucosa. Expansion of the ten Hove study to ulcerative colitis and other chronic inflammatory conditions should provide rather interesting answers to the questions and speculation raised in this commentary.

Gut 2002;50:148-149 


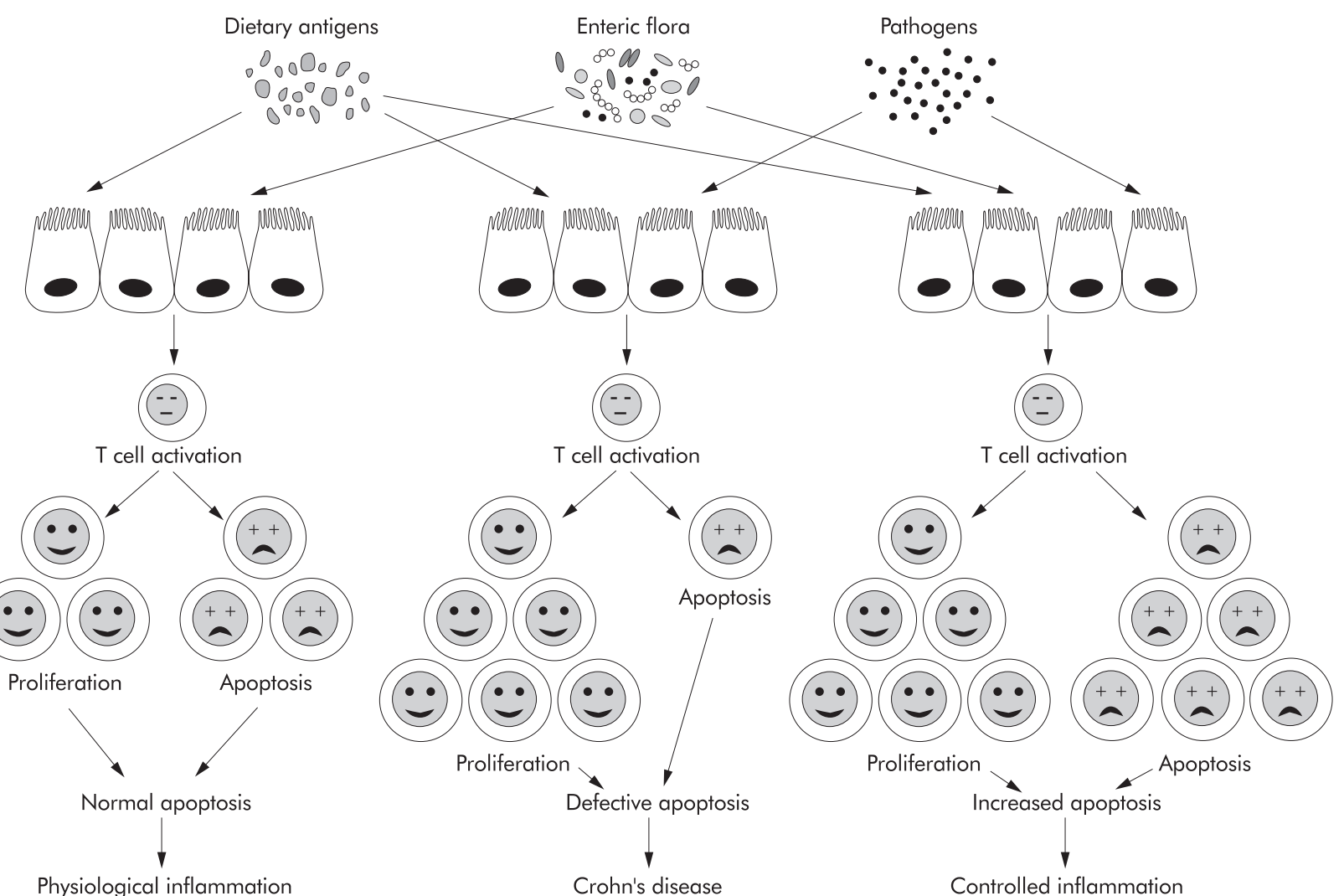

Figure 1 Schematic representation of the various outcomes resulting from the balance between T cell proliferation and apoptosis (cell death) in the normal intestinal mucosa, or imbalance during chronic intestinal inflammation. In health, mucosal T cell proliferation induced by dietary and enteric flora antigens is counterbalanced by a baseline (normal) degree of apoptosis resulting in the low degree of physiological inflammation found in the normal gut. In Crohn's disease there is increased T cell proliferation induced by still undefined stimuli derived from bacterial antigens, food, and possibly unrecognised pathogens. At the same time, T cells die less because of their resistance to apoptosis. This defective apoptosis results in inappropriate T cell accumulation that fosters inflammation. If the degree of T cell apoptosis is increased in Crohn's disease, an effect apparently mediated by infliximab, a new balance is established between the increased rate of proliferation due to inflammation and the increased rate of apoptosis. A state of controlled inflammation is then established that is translated into clinical improvement, and later on decreased cellularity of the mucosa.

\section{Authors' affiliations}

A Sturm, C Fiocchi, Division of Gastroenterology, University Hospitals of Cleveland and Case Western Reserve University School of Medicine, 10900 Euclid Avenue, BRB 425, Cleveland, Ohio 44106-4952, USA; cxf18@po.cwru.edu

\section{REFERENCES}

1 Elliott MJ, Maini RN, Feldmann M, et al. Randomised double-blind comparison of chimeric monoclonal antibody to tumour necrosis factor $\alpha$ (cA2) versus placebo in rheumatoid arthritis. Lancet 1994;344: 1 105-10

2 Targan SR, Hanauer SB, van Deventer SJH et al. A short-term study of chimeric monoclonal antibody cA2 to tumor necrosis factor $\alpha$ for Crohn's disease. N Engl J Med 1997;337: 1029-35

3 ten Hove T, van Montfrans C, Peppelenbosch $M P$, et al. Infliximab treatment induces apoptosis of lamina propria T lymphocytes in Crohn's disease. Gut 2002;50:206-21 1 .
4 Feldmann M Maini RN. Anti-TNF $\alpha$ therapy of rheumatoid arthritis: what have we learned? Annu Rev Immunol 2001;19:163-96.

5 Papadakis KA, Targan SR. Tumor necrosis factor: biology and therapeutic inhibitors. Gastroenterology 2000;1 19:1 148-57.

6 Baert FJ, D'Haens GR, Peeters M, et al. Tumor necrosis factor $\alpha$ antibody (infliximab) therapy profoundly down-regulates the inflammation in Crohn's ileocolitis. Gastroenterology 1999;116:22-8.

7 Thompson CB. Apoptosis in the pathogenesis and treatment of disease. Science and treatment of disease

8 Sprent J, Tough DF. T cell death and memory. Science 2001;293:245-58.

9 Boirivant M, Marini M, Di Felice G, et al. Lamina propria T cells in Crohn's disease and other gastrointestinal inflammation show defective CD2 pathway-induced apoptosis. Gastroenterology 1999; 1 16:557-65.

10 Ina K, Itoh J, Fukushima K, et al. Resistance of Crohn's disease T-cells to multiple apoptotic stimuli is associated with a $\mathrm{Bcl}-2 / \mathrm{Bax}$ mucosal imbalance. J Immunol 1999;163:1081-90.

11 Itoh J, delaMotte C, Strong SA, et al. Decreased Bax expression by mucosal T-cells favours resistance to apoptosis in Crohn's disease (CD). Gut 2001;49:35-41.

12 Fuss IJ, Marth T, Neurath MF, et al. Anti-interleukin-12 treatment regulates apoptosis of Th1 T cells in experimental colitis in mice. Gastroenterology 1999;117:107888.

13 Atreya R, Mudter J, Finotto S, et al. Blockade of interleukin 6 trans signaling suppresses T-cell resistance against apoptosis in chronic intestinal inflammation: evidence in Crohn disease and experimental colitis in vivo. Nat Med 2000;6:583-8.

14 Wittig BM, Johansson B, Zoller $M$, et al. Abrogation of experimental colitis correlates with increased apoptosis in mice deficient for CD44 variant exon 7 (CD44v7). J Exp Med 2000; 12:2053-63.

15 Cornillie F, Shealy D, D'Haens G, et al. Infliximab induces potent anti-inflammatory and local immunomodulatory activity but no systemic immune suppression in patients with Crohn's disease. Aliment Pharmacol Ther 2001;15:463-73.

16 Sands BE, Tremaine WJ, Sandborm WJ, et al. Infliximab in the treatment of severe, steroid-refractory ulcerative colitis: a pilot study. Inflamm Bowel Dis 2001;7:83-8. 